Supporting Information

\title{
A first molecular dynamics study for modeling the microstructure and mechanical behavior of Si nanopillars during lithiation
}

Fei Shuang and Katerina E. Aifantis*

Department of Mechanical and Aerospace Engineering, University of Florida

PO BOX 116250, Gainesville, FL, 32611, USA

*Corresponding Author

E-mail: kaifantis@ufl.edu

Phone: (352) 392-6227 
1 Predefined lithiation profiles
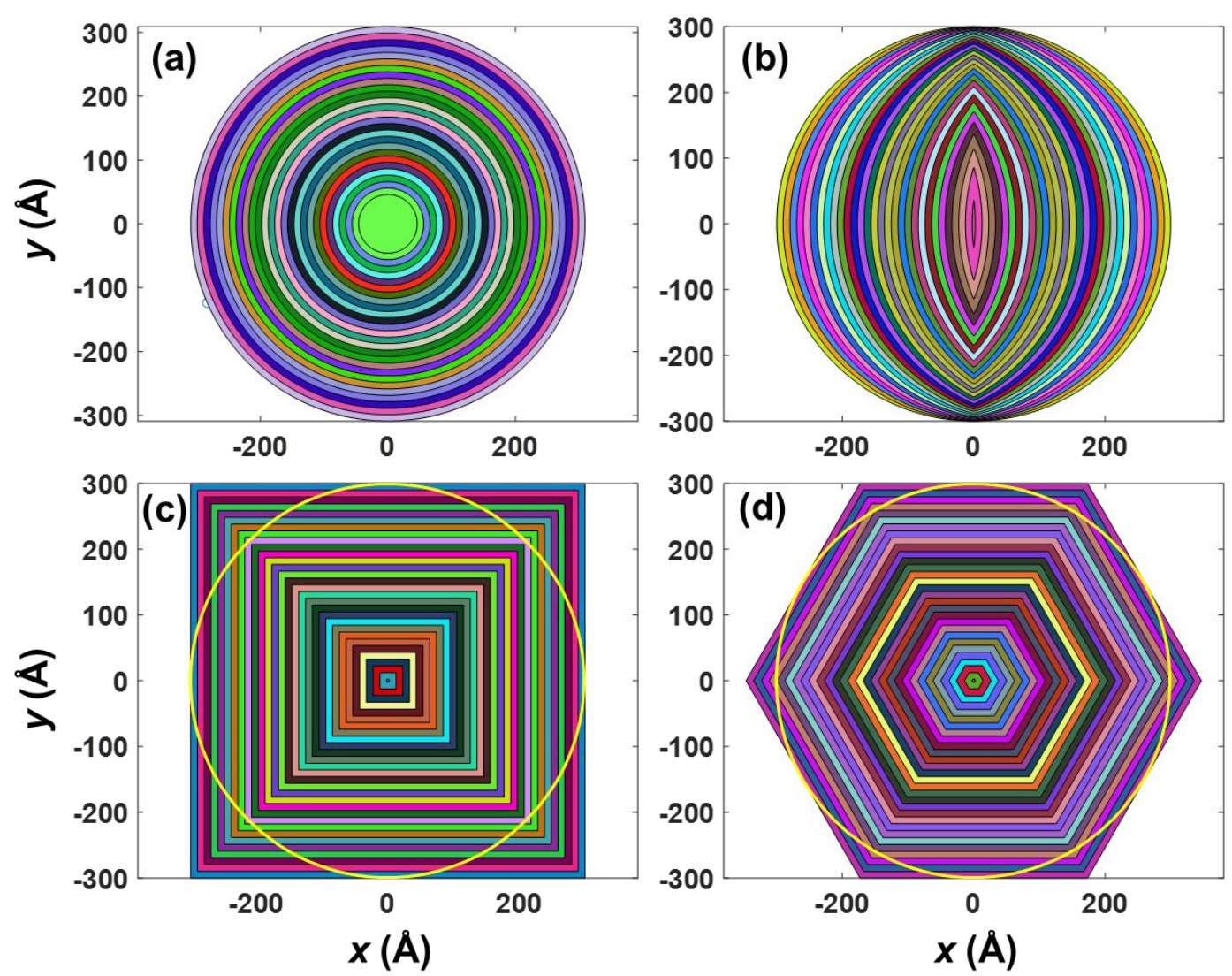

Figure S1. Predefined lithiation layers for a-SiNP and three c-SiNPs with different axial orientations. (a) a-SiNP, (b) $<110>$ c-SiNP, (c) $<100>$ c-SiNP and (d) $<111>$ c-SiNP. The yellow lines represent the initial SiNPs.

2 Simulation results with and without NVE dynamics relaxation

Figure $\mathrm{S} 2$ shows that NVE dynamics relaxation is of great importance for determining the size and morphologies of lithiated SiNPs. 

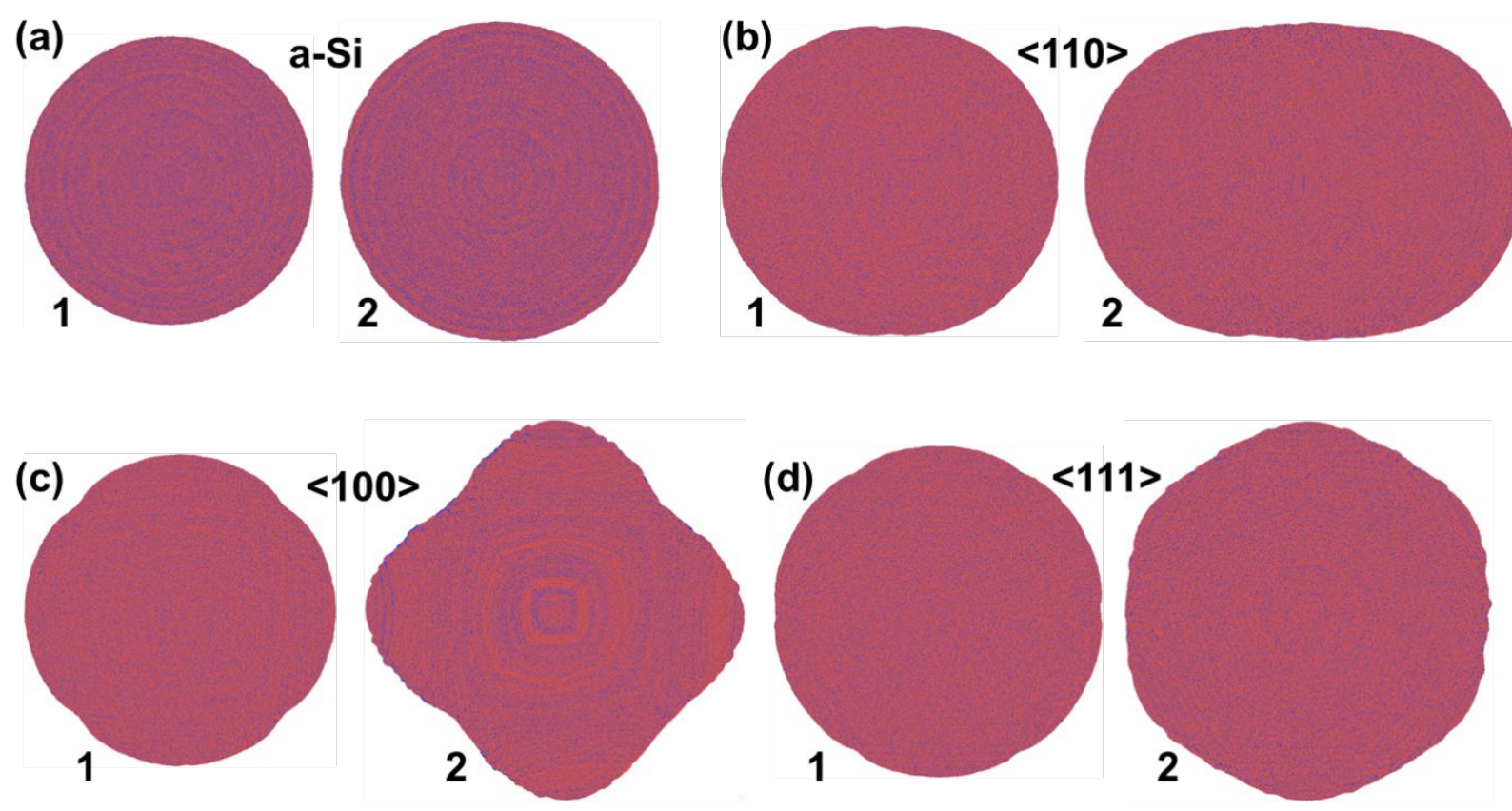

Figure S2. Morphologies of a-SiNP and c-SiNPs with and without NVE dynamics relaxation: (a) a-SiNP, (b) $<110>$ c-SiNP, (c) $<100>$ c-SiNP and (d) $<111>$ c-SiNP. All SiNPs have diameter of $30.0 \mathrm{~nm}$ before lithiation. $\left(\mathrm{a}_{1}-\mathrm{d}_{1}\right)$ correspond to the morphologies without NVE dynamics relaxation and $\left(\mathrm{a}_{2}-\mathrm{d}_{2}\right)$ correspond to the morphologies with NVE dynamics relaxation.

3 Atomic stress and strain calculation

The stress tensor for each atom is calculated by LAMMPS ${ }^{1}$ :

$$
S_{a b}=-m v_{a} v_{b}-W_{a b}
$$

where $a$ and $b$ takes on values $x, y, z$ to generate the six stress, $m$ is the atomic mass and $v$ is the atomic velocity. The first term in Equation (S1) is a kinetic energy contribution, and the second term $W_{a b}$ is the virial contribution due to intra and intermolecular interactions. The atomic stress can be obtained by:

$$
\sigma_{a b}=\frac{S_{a b}}{\Omega}
$$


where $\Omega$ is the atomic volume which can be calculated by the Voronoi analysis in the OVITO software $^{2}$. Note that the atomic volume is ill-defined for the atoms at the outer surface, such that the atomic stresses are not available for the outer surface as seen in Fig. S3. The stress components in a cylindrical coordinate system can be calculated by coordinate transformation. The hoop stress is obtained as:

$$
\sigma_{\theta}=\sigma_{x} \sin ^{2} \theta+\sigma_{y} \cos ^{2} \theta-2 \tau_{x y} \sin \theta \cos \theta
$$

The radial stress is:

$$
\sigma_{r}=\sigma_{x} \cos ^{2} \theta+\sigma_{y} \sin ^{2} \theta+2 \tau_{x y} \sin \theta \cos \theta
$$

And the axial stress is:

$$
\sigma_{z}=\sigma_{z}
$$

where $\theta$ is the angle in cylindrical coordinate system.

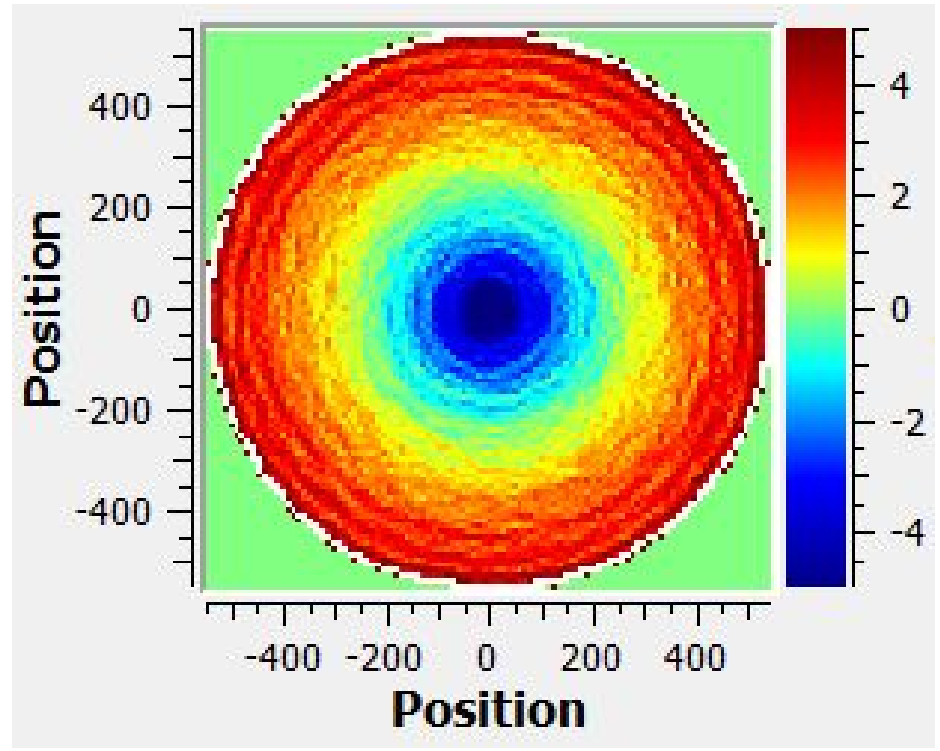

Figure S3. Plot of hoop stress for a-SiNP with initial radius of $30.0 \mathrm{~nm}$ by using $100 \times 100$ bins.

The von Mises local shear strain is used to measure the inelastic deformation at the atomic scale. The calculation is done by the software OVITO $^{2}$ based on finite-strain theory: 


$$
\begin{gathered}
\eta=\sqrt{E_{x y}^{2}+E_{x z}^{2}+E_{y z}^{2}+1 / 6\left(\left(E_{x x}-E_{y y}\right)^{2}+\left(E_{x x}-E_{z z}\right)^{2}+\left(E_{y y}-E_{y y}\right)^{2}\right)} \\
\mathbf{E}=1 / 2\left(\mathbf{F}^{T} \mathbf{F}-\mathbf{I}\right)
\end{gathered}
$$

where $\mathbf{E}$ is the Green-Lagrangian strain tensor and $\mathbf{F}$ is the deformation gradient tensor.

4 Evolution of the outer radius of hollow a-SiNPs during lithiation

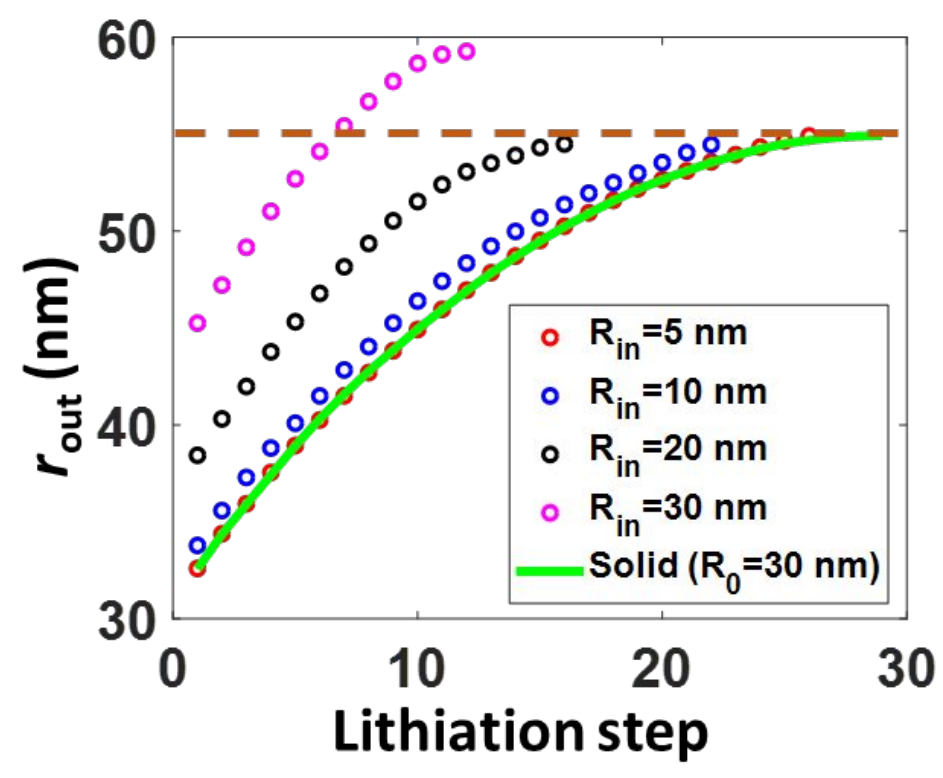

Figure S4. Evolution of the outer radius $\left(r_{\text {out }}\right)$ of hollow a-SiNPs during lithiation 
5 Stress field near three dislocations in the c-Si core

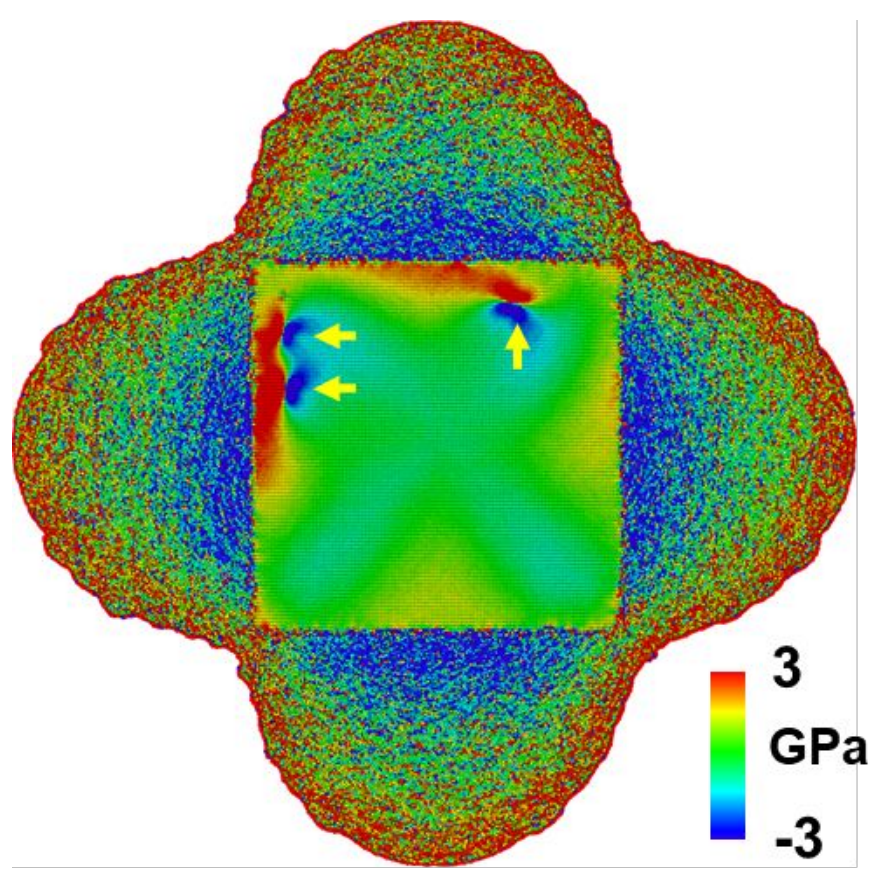

Figure S5. Stress field near three dislocations in the c-Si core.

\section{Reference}

(1) Plimpton, S. Fast Parallel Algorithms for Short - Range Molecular Dynamics. J. Comput. Phys. 1995, 117, 1-19.

(2) Stukowski, A. Visualization and Analysis of Atomistic Simulation Data with OVITO-the Open Visualization Tool. Model. Simul. Mater. Sci. Eng. 2010, 18 (1), 015012. 